\title{
Transition Decomposition of Quantum Mechanical Evolution
}

\author{
Y. Strauss ${ }^{1}$, J. Silman ${ }^{2,3}$, S. Machnes ${ }^{2,4}$, and L.P. Horwitz ${ }^{2,5,6}$ \\ ${ }^{1}$ Einstein Institute of Mathematics, Edmond J. Safra campus, \\ The Hebrew University of Jerusalem, Jerusalem 91904, Israel \\ ${ }^{2}$ School of Physics and Astronomy, Raymond and Beverly Sackler \\ Faculty of Exact Sciences, Tel-Aviv University, Tel-Aviv 69978, Israel \\ ${ }^{3}$ Laboratoire d'Information Quantique, Université Libre de Bruxelles, 1050 Bruxelles, Belgium \\ ${ }^{4}$ Institut für Theoretische Physik, Universität Ulm, 89069 Ulm, Germany \\ ${ }^{5}$ Physics Department of Physics, Bar-Ilan University, Ramat-Gan 52900, Israel \\ ${ }^{6}$ Department of Physics, The Ariel University Center of Samaria, Ariel 40700, Israel
}

\begin{abstract}
We show that the existence of the family of self-adjoint Lyapunov operators introduced in [J. Math. Phys. 51, 022104 (2010)] allows for the decomposition of the state of a quantum mechanical system into two parts: A past time asymptote, which is asymptotic to the state of the system at $t \rightarrow-\infty$ and vanishes at $t \rightarrow \infty$, and a future time asymptote, which is asymptotic to the state of the system at $t \rightarrow \infty$ and vanishes at $t \rightarrow-\infty$. We demonstrate the usefulness of this decomposition for the description of resonance phenomena by considering the resonance scattering of a particle off a square barrier potential. We show that the past time asymptote captures the behavior of the resonance. In particular, it exhibits the expected exponential decay law and spatial probability distribution.
\end{abstract}

\section{INTRODUCTION}

In standard non-relativistic quantum mechanics time enters as a parameter, external to the quantum system being investigated, and as such is not a dynamical variable associated in any way with the system's dynamics. However, there are many cases, such as experiments measuring the time of arrival of particles at a detector, the decay time of an unstable quantum system etc., which call for a more dynamical point of view with respect to time. One approach to this problem consists of the construction of time operators through the use of covariant positive operator valued measures (POVMs) 1, 2]. Since the time operators built via the use of POVMs are in general maximally symmetric, non-self-adjoint operators, the construction of these objects bypasses an old theorem of Pauli [3], stating that there does not exist a self-adjoint time operator $T$ canonically conjugate to a Hamiltonian $H$ whose spectrum is semibounded, such that the pair $T$ and $H$ forms together an imprimitivity system [4]. However, in comparison to standard self-adjoint quantum observables these operators present some difficulties, e.g., in the relation between the algebra they generate and their spectral representations.

The dynamical role of time within the framework of standard quantum mechanics has recently been considered in [5, 6] through the construction of a family of self-adjoint Lyapunov operators. Throughout this text we define a Lyapunov operator as a self-adjoint operator whose expectation value is monotonically decreasing in time. More precisely, let $\mathcal{H}$ be a Hilbert space corresponding to a given quantum mechanical system, let $H$ be a self-adjoint Hamiltonian generating its evolution, and let $|\psi(t)\rangle=U(t)|\psi\rangle=\exp (-i H t)|\psi\rangle$ be the state of the system at time $t$. Define the trajectory $\Psi_{\psi}$, corresponding to an initial state $|\psi\rangle \in \mathcal{H}$, as the set of states

$$
\Psi_{\psi}:=\{|\psi(t)\rangle\}_{t \in \mathbb{R}^{+}}=\{U(t)|\psi\rangle\}_{t \in \mathbb{R}^{+}} .
$$

Then the definition of a Lyapunov operator is as follows [5]:

Definition 1: Let $M$ be a bounded self-adjoint operator on $\mathcal{H}$. Let $\Psi_{\psi}$ be a trajectory corresponding to an arbitrarily chosen initial state $|\psi\rangle \in \mathcal{H}$. Denote by $\left.M\left(\Psi_{\psi}\right)=\{\langle\varphi|M| \varphi\rangle|| \varphi\rangle \in \Psi_{\psi}\right\}$ the collection of all expectation values of $M$ for normalized states in $\Psi_{\psi}$. Then $M$ is a forward Lyapunov operator if the mapping $\tau_{M, \psi}: \mathbb{R}^{+} \mapsto M\left(\Psi_{\psi}\right)$ defined by

$$
\tau_{M, \psi}(t)=\langle\psi(t)|M| \psi(t)\rangle
$$

is monotonically decreasing in time.

Remark 1: If in the definition above we require that $\tau_{M, \psi}$ be monotonically increasing instead of monotonically decreasing we also obtain a valid definition of a Lyapunov operator. The requirement that $\tau_{M, \psi}$ is monotonically decreasing is made purely for the sake of convenience. 
It follows from this definition that a Lyapunov operator allows for the temporal ordering of states in any trajectory $\Psi_{\psi}$ according to the ordering of the expectation values in $M\left(\Psi_{\psi}\right)$, thereby introducing temporal ordering into the Hilbert space $\mathcal{H}$ of any problem for which such an operator can be constructed.

The construction of Lyapunov operators constitutes a somewhat conservative approach to the problem of time in quantum mechanics. In general, Lyapunov operators indicate the direction of flow of time in a system but do not serve as time operators and are not intended for answering questions of direct time measurements. Rather, the construction of Lyapunov operators, and in particular the family of Lyapunov operators introduced in [5, 6], was carried out with a different goal in mind. The temporal ordering introduced into the Hilbert space is a fundamental property of a quantum mechanical problem admitting Lyapunov operators. A question then arises as to the possible implications this property may have on the description of the dynamics of the system, i.e. is there a way to 'inject' the direction of time ordering introduced by the Lyapunov operator into the description of the dynamics of a quantum system in such a way that certain processes in the evolution of the system obtain simple descriptions amenable to thorough analyses? The study of such consequences of the existence of a Lyapunov operator has been addressed to some extent in [5, 6]. In the present paper we continue this line of investigation, and demonstrate the beginning of an affirmative answer to the question raised above. Specifically, we show that the existence of the family of Lyapunov operators introduced in [5, 6] leads to a certain description of the scattering process, which we shall term the transition decomposition, that is particularly useful for treating the evolution of scattering resonances.

\section{LYAPUNOV OPERATORS}

In this section we state some key results from [5, 6], which will provide the basis for the all that follows. The following theorem is an adaptation of results stated in [5, 6] :

Theorem 1: Let $\mathcal{H}$ be the Hilbert space representing some quantum mechanical system, let $H$ be its Hamiltonian, and let the spectrum of $H$ be $\mathbb{R}^{+}$, absolutely continuous, and uniformly degenerate. Then for any choice of generalized eigenbasis $|E, \lambda\rangle$ of $H, \lambda$ being the degeneracy index, the operator

$$
M=-\frac{1}{2 \pi i} \sum_{\lambda} \int_{0}^{\infty} d E \int_{0}^{\infty} d E^{\prime}|E, \lambda\rangle \frac{1}{E-E^{\prime}+i 0^{+}}\left\langle E^{\prime}, \lambda\right|,
$$

where the summation over $\lambda$ may also stand for integration, is a Lyapunov operator in the sense of Definition 1, and, moreover, for any state $|\psi\rangle \in \mathcal{H}$

$$
\lim _{t \rightarrow \infty}\langle\psi(t)|M| \psi(t)\rangle=0, \quad \lim _{t \rightarrow-\infty}\langle\psi(t)|M| \psi(t)\rangle=1
$$

Remark 2: In this paper we shall consider only Hamiltonians for which the spectrum is $\mathbb{R}^{+}$, absolutely continuous, and uniformly degenerate. The results below apply also to the more general case of Hamiltonians for which the absolutely continuous spectrum is $\mathbb{R}^{+}$and is uniformly degenerate. In such cases the constructions below hold in the subspace $\mathcal{H}_{a c}$.

Remark 3: Note that each complete set of generalized eigentates of $H$ has associated with it a different Lypaunov operator, and as such Theorem 1 represents a prescription for the construction of a family of self-adjoint Lyapunov operators. In particular, this still holds true for two sets that differ only by an energy dependent phase. When discussing general properties of the set of Lypaunov operators defined above, we shall often refer to $M$ without specifying the underlying complete set of generalized eigenstates.

Remark 4: The above family of self-adjoint Lyapunov operators has recently been generalized in [7].

Remark 5: Throughout this paper we employ natural units, i.e. $\hbar=c=1$.

The following corollary to Theorem 1 is crucial for what follows.

Corollary 1: Let $\Lambda:=M^{1 / 2}$. Then for any state $|\psi\rangle \in \mathcal{H}$

$$
\lim _{t \rightarrow \infty}\langle\psi(t)|\Lambda| \psi(t)\rangle=0, \quad \lim _{t \rightarrow-\infty}\langle\psi(t)|\Lambda| \psi(t)\rangle=1 .
$$




\section{THE TRANSITION DECOMPOSITION}

In this section we present a decomposition of the wave-function into two components - one that is asymptotic to the state of the system in the far past and vanishes in the far future, and one that is asymptotic to the state of the system in the far future and vanishes in the far past - and a corresponding decomposition for operators when working in the Heisenberg picture. The transition over time between the two asymptotic components suggests the possibility that this decomposition yields an appropriate description of transient phenomena in the evolution of a quantum system. This expectation is realized when we apply the decomposition to scattering problems, where it will be seen to afford a highly useful description of the evolution of scattering resonances. This task is taken up in the next section.

Let $|\psi(t)\rangle=U(t)|\psi\rangle,|\psi\rangle \in \mathcal{H}, t \in \mathbb{R}$. At any moment in time $t$ express $\psi(t)$ as the sum of two components as follows:

$$
|\psi(t)\rangle=\left|\psi_{b}(t)\right\rangle+\left|\psi_{f}(t)\right\rangle
$$

where

$$
\left|\psi_{b}(t)\right\rangle:=\Lambda|\psi(t)\rangle, \quad\left|\psi_{f}(t)\right\rangle:=(I-\Lambda)|\psi(t)\rangle
$$

The limits below are then an immediate consequence of Theorem 1 and Corollary 1:

$$
\begin{array}{rlrl}
\lim _{t \rightarrow-\infty} \||\psi(t)\rangle-\left|\psi_{b}(t)\right\rangle \| & =0, & & \lim _{t \rightarrow \infty} \|\left|\psi_{b}(t)\right\rangle \|=0, \\
\lim _{t \rightarrow-\infty} \|\left|\psi_{f}(t)\right\rangle \| & =0, & \lim _{t \rightarrow \infty} \||\psi(t)\rangle-\left|\psi_{f}(t)\right\rangle \|=0 .
\end{array}
$$

Eqs. (6), (8), and (9) imply that $|\psi(t)\rangle$ can be decomposed into a sum of two components, $\left|\psi_{b}(t)\right\rangle$ and $\left|\psi_{f}(t)\right\rangle$, such that $\left|\psi_{b}(t)\right\rangle$ vanishes in the future time asymptote and is asymptotic to $|\psi(t)\rangle$ in the past time asymptote, and $\left|\psi_{f}(t)\right\rangle$ vanishes in the past time asymptote and is asymptotic to $|\psi(t)\rangle$ in the future time asymptote. We refer to $\left|\psi_{b}(t)\right\rangle$ as the backward asymptotic component and to $\left|\psi_{f}(t)\right\rangle$ as the forward asymptotic component of $|\psi(t)\rangle$. Since the decomposition here is of the evolving state $|\psi(t)\rangle$, we call the decomposition in Eq. (6) the transition decomposition in the Schrödinger picture. In this decomposition the evolution of $|\psi(t)\rangle$ is represented as a transition from its backward asymptotic component to its forward asymptotic component.

The transition decomposition in the Schrödinger picture, Eq. (6), gives rise to a corresponding decomposition in the Heisenberg picture. Let $X$ be a self-adjoint operator representing some physical observable and let $X(t)=$ $U^{\dagger}(t) X U(t)$ be its Heisenberg evolution. Consider the expectation value of $X(t)$ for some arbitrary state $|\psi\rangle \in \mathcal{H}$ and apply the decomposition in Eq. (6). We have

$$
\begin{gathered}
\langle\psi|X(t)| \psi\rangle=\langle\psi(t)|X| \psi(t)\rangle=\left\langle\psi_{b}(t)+\psi_{f}(t)|X| \psi_{b}(t)+\psi_{f}(t)\right\rangle \\
=\left\langle\psi_{b}(t)|X| \psi_{b}(t)\right\rangle+\left(\left\langle\psi_{b}(t)|X| \psi_{f}(t)\right\rangle+\left\langle\psi_{f}(t)|X| \psi_{b}(t)\right\rangle\right)+\left\langle\psi_{f}(t)|X| \psi_{f}(t)\right\rangle .
\end{gathered}
$$

Define now the following decomposition of $X$, which will be seen below to correspond to the three different terms on the right-hand side of Eq. (10),

$$
X:=X_{b}+X_{t r}+X_{f}
$$

with

$$
X_{b}:=\Lambda X \Lambda, \quad X_{t r}:=\Lambda X(I-\Lambda)+(I-\Lambda) X \Lambda, \quad X_{f}:=(I-\Lambda) X(I-\Lambda) .
$$

Indeed, if $X_{b}(t), X_{t r}(t)$, and $X_{f}(t)$, respectively, are the Heisenberg evolutions of $X_{b}, X_{t r}$, and $X_{f}$, so that

$$
X(t)=X_{b}(t)+X_{t r}(t)+X_{f}(t),
$$

then

$$
\begin{gathered}
\left\langle\psi\left|X_{b}(t)\right| \psi\right\rangle=\left\langle\psi(t)\left|X_{b}\right| \psi(t)\right\rangle=\langle\psi(t)|\Lambda X \Lambda| \psi(t)\rangle=\left\langle\psi_{b}(t)|X| \psi_{b}(t)\right\rangle, \\
\left\langle\psi\left|X_{t r}(t)\right| \psi\right\rangle=\left\langle\psi(t)\left|X_{t r}\right| \psi(t)\right\rangle=\langle\psi(t)|\Lambda X(I-\Lambda)+(I-\Lambda) X \Lambda| \psi(t)\rangle \\
=\left\langle\psi_{b}(t)|X| \psi_{f}(t)\right\rangle+\left\langle\psi_{f}(t)|X| \psi_{b}(t)\right\rangle,
\end{gathered}
$$




$$
\left\langle\psi\left|X_{f}(t)\right| \psi\right\rangle=\left\langle\psi(t)\left|X_{f}\right| \psi(t)\right\rangle=\langle\psi(t)|(I-\Lambda) X(I-\Lambda)| \psi(t)\rangle=\left\langle\psi_{f}(t)|X| \psi_{f}(t)\right\rangle .
$$

Using the limits of $\left|\psi_{b}(t)\right\rangle$ and $\left|\psi_{f}(t)\right\rangle$, Eqs. (8) and (9), we obtain that the components of $X(t)$ satisfy the following limits

$$
\begin{aligned}
\lim _{t \rightarrow-\infty}\left(X(t)-X_{b}(t)\right) & =0, & & \lim _{t \rightarrow \infty} X_{b}(t)=0, \\
\lim _{t \rightarrow-\infty} X_{t r}(t) & =0, & & \lim _{t \rightarrow \infty} X_{t r}(t)=0, \\
\lim _{t \rightarrow-\infty} X_{f}(t) & =0, & & \lim _{t \rightarrow \infty}\left(X(t)-X_{f}(t)\right)=0 .
\end{aligned}
$$

Eq. (13) provides the transition decomposition in the Heisenberg picture. The observable $X(t)$ decomposes into a sum of three components, $X_{b}(t), X_{t r}(t)$, and $X_{f}(t)$, such that $X_{b}(t)$ vanishes in the future time asymptote and is asymptotic to $X(t)$ in the past time asymptote, $X_{f}(t)$ vanishes in the past time asymptote and is asymptotic to $X(t)$ in the future time asymptote, and $X_{t r}(t)$ is transient and vanishes both in the past and in the future time asymptotes. The evolution of $X(t)$ is represented as a transition from $X_{b}(t)$ in the backward asymptote to $X_{f}(t)$ in the forward asymptote. Accordingly, $X_{b}(t)$ will be termed the backward asymptotic transition observable, $X_{f}(t)$ will be termed the forward asymptotic transition observable, and $X_{t r}(t)$ will be termed the transient observable.

\section{APPLICATION TO SCATTERING PROBLEMS}

In this section we apply the transition decomposition to a quantum mechanical scattering problem. In particular, we find that this decomposition is especially useful for the description of scattering resonances. Specifically, we shall apply the transition decomposition to the evolution of approximate resonance states defined in the context of the formalism of the semigroup decomposition of resonance evolution [8, 9]. This formalism, developed in recent years out of efforts to adapt the Lax-Phillips scattering theory [10] to the description of the evolution of scattering resonances in quantum mechanics [11], is a mathematical framework serving as a basis for a time-dependent theory of resonances in quantum mechanical scattering problems [12]. We give here a short presentation of the simplest form of this framework that is sufficient for our purposes.

Recall that Theorem 1 defines a large class of Lypaunov operators in the sense that for any complete set of generalized eigenstates of the Hamiltonian there corresponds a different Lyapunov operator. It follows that each choice of Lyapunov operator has associated with it its own transition decomposition. We would like to apply the transition decomposition to scattering problems. For such problems there are at least two distinguished energy representations for the Hamiltonian, i.e. the incoming and outgoing energy representations, and we must choose which of the two to employ. The incoming and outgoing energy representations are defined in terms of the incoming and outgoing solutions of the Lipmann-Schwinger equation. Let $\left|E^{+}, \lambda\right\rangle$ and $\left|E^{-}, \lambda\right\rangle$ be the incoming and outgoing solutions of the Lipmann-Schwinger equation satisfying $H\left|E^{ \pm}, \lambda\right\rangle=E\left|E^{ \pm}, \lambda\right\rangle$, where $\lambda$ stands for the degeneracy indices of the energy spectrum. (The degeneracy is assumed to be uniform over the energy spectrum.) The outgoing Lyapunov operator $M^{+}$is defined as

$$
M^{+}=-\frac{1}{2 \pi i} \sum_{\lambda} \int_{0}^{\infty} d E \int_{0}^{\infty} d E^{\prime}\left|E^{-}, \lambda\right\rangle \frac{1}{E-E^{\prime}+i 0^{+}}\left\langle E^{\prime-}, \lambda\right|,
$$

and gives rise to an outgoing transition decomposition in the Schrödinger or Heisenberg pictures. Defining $\Lambda^{+}:=$ $M^{+1 / 2}$, the outgoing transition decomposition in the Schrödinger picture is given by

$$
|\psi(t)\rangle=\left|\psi_{b}^{+}(t)\right\rangle+\left|\psi_{f}^{+}(t)\right\rangle
$$

while for an observable $X$ defined on $\mathcal{H}$, in the outgoing transition decomposition in the Heisenberg picture, we have

$$
X=X_{b}^{+}+X_{t r}^{+}+X_{f}^{+}
$$

with

$$
X_{b}^{+}=\Lambda^{+} X \Lambda^{+}, \quad X_{t r}^{+}=\Lambda^{+} X\left(I-\Lambda^{+}\right)+\left(I-\Lambda^{+}\right) X \Lambda^{+}, \quad X_{f}^{+}=\left(I-\Lambda^{+}\right) X\left(I-\Lambda^{+}\right)
$$

To proceed we assume the following: 
(i) Let $\mathcal{H}$ be a Hilbert space corresponding to a given quantum mechanical scattering problem. A self-adjoint 'free' unperturbed Hamiltonian $H_{0}$ and a self-adjoint perturbed Hamiltonian $H$ are defined on $\mathcal{H}$ and form a complete scattering system, i.e., we assume that the Møller wave operators $\Omega^{ \pm}\left(H_{0}, H\right)$ exist and are complete.

(ii) We assume that the (absolutely) continuous spectrum of $H$ is uniformly degenerate. To simplify matters we assume that this degeneracy is one.

(iii) The $S$-matrix in the the energy representation, denoted by $\tilde{\mathcal{S}}(E)$, is the boundary value of a function $\mathcal{S}(z)$ analytic in some strip above the positive real axis and having an analytic continuation across the cut on the positive real energy axis into some simply-connected region $\Sigma$ below the real axis in which it has a single, simple resonance pole at the point $z=\mu$ with $\operatorname{Im} \mu<0$.

It is shown in [8, 9] that there exists a dense set $\Xi \subset \mathcal{H}$ and a well defined state $\left|\psi_{\mu}^{a p p}\right\rangle \in \mathcal{H}$ such that for any states $|\varphi\rangle \in \Xi$ and $|\psi\rangle \in \mathcal{H}$ the above assumptions lead to a decomposition, induced by the pole of the $S$-matrix at $z=\mu$, of matrix elements of the evolution $U(t)$ generated by $H$, having the form

$$
\langle\varphi \mid U(t) \psi\rangle=B(\varphi, \psi, \mu ; t)+\alpha(\varphi, \mu)\left\langle\psi_{\mu}^{a p p} \mid \psi\right\rangle e^{-i \mu t}, \quad t \geq 0
$$

The second term of the decomposition on the right-hand side of Eq. (24) exhibits the typical decay behavior of a resonance. This term is the semigroup term, or resonance term. The term $B(\varphi, \psi, \mu ; t)$ in Eq. (24) is the so-called background term. The state $\left|\psi_{\mu}^{\text {app }}\right\rangle$ appearing in the second term in Eq. (24) is called the approximate resonance state. Note that if the state $|\psi\rangle$ on the left-hand side of Eq. (24) is chosen to be orthogonal to $\left|\psi_{\mu}^{a p p}\right\rangle$ then the resonance term in that equation vanishes. The reference to $\left|\psi_{\mu}^{a p p}\right\rangle$ as an approximate resonance state stems from the fact that it can be shown that there is no choice of $|\varphi\rangle$ and $|\psi\rangle$ in the matrix element $\langle\varphi \mid U(t) \psi\rangle$ for which the background term $B(\varphi, \psi, \mu ; t)$ disappears [8]. Indeed, the Schrödinger evolution of a closed system does not allow for an exponential decay law for the survival probability [13] and deviations, such as the Zeno effect for short times are inevitable [14].

An explicit expression for the approximate resonance state $\left|\psi_{\mu}^{a p p}\right\rangle$ is given by [8, 9, 15]

$$
\left|\psi_{\mu}^{a p p}\right\rangle=\frac{1}{2 \pi i} \int_{\mathbb{R}^{+}} d E \frac{1}{E-\mu}\left|E^{-}\right\rangle .
$$

This expression is obtained under the assumption that there is only a single resonance pole of the $S$-matrix below the positive real axis in the region $\Sigma$. In the case that $\Sigma$ contains multiple resonance poles of the $S$-matrix Eq. 25 provides only a zeroth order approximate resonance state [9]. Throughout the rest of the paper we only consider approximate resonance states given by Eq. (25). This restriction pertains also to the example worked out below for which the $S$-matrix possesses multiple resonance poles. Hence, all states calculated there are of zeroth order. The restriction to zeroth order approximate resonance states is made for the sake of simplicity and clarity of exposition and it should be emphasized that there is no a priori difficulty in working with higher order approximate resonance states.

Applying the outgoing transition decomposition in Eq. (22) to the expectation value of an observable $X(t)$ in the state $\left|\widetilde{\psi}_{\mu}^{a p p}\right\rangle:=\|\left|\psi_{\mu}^{a p p}\right\rangle \|^{-1}\left|\psi_{\mu}^{a p p}\right\rangle$, we get

$$
\left\langle\tilde{\psi}_{\mu}^{a p p}|X(t)| \tilde{\psi}_{\mu}^{a p p}\right\rangle=\left\langle\tilde{\psi}_{\mu}^{a p p}\left|X_{b}^{+}(t)\right| \tilde{\psi}_{\mu}^{a p p}\right\rangle+\left\langle\tilde{\psi}_{\mu}^{a p p}\left|X_{t r}^{+}(t)\right| \tilde{\psi}_{\mu}^{a p p}\right\rangle+\left\langle\tilde{\psi}_{\mu}^{a p p}\left|X_{f}^{+}(t)\right| \tilde{\psi}_{\mu}^{a p p}\right\rangle .
$$

We shall use this decomposition to represent the evolution of a particular resonance in a simple one-dimensional scattering problem. The model we consider is the scattering along the half-line $\mathbb{R}^{+}$off a square barrier potential. Thus, we consider a free Hamiltonian $H_{0}=-\frac{1}{2 m} \partial_{x}^{2}$ acting on $L^{2}\left(\mathbb{R}^{+}\right.$) (where $H_{0}$ is taken to be the self-adjoint extension in $L^{2}\left(\mathbb{R}^{+}\right)$of $-\frac{1}{2 m} \partial_{x}^{2}$ from its original domain of definition $\left.\mathcal{D}\left(\partial_{x}^{2}\right)=\left\{\phi(x) \mid \phi(x) \in W_{2}^{2}\left(\mathbb{R}^{+}\right), \phi(0)=0\right\}\right)$ and a full Hamiltonian $H=H_{0}+V$ with $V$ a multiplicative operator $(V \psi)(x)=V(x) \psi(x)$ such that

$$
V(x)= \begin{cases}0, & 0<x<a \\ V_{0}, & a \leq x \leq b \\ 0, & b<x\end{cases}
$$

where $0<a<b$ and $V_{0}>0$. In this case there are no bound state solutions of the eigenvalue problem for $H$ and the (absolutely) continuous spectrum of $H$ is $\mathbb{R}^{+}$. In order to find the scattering states and calculate the $S$-matrix one solves the eigenvalue problem for the continuous spectrum generalized eigenfunctions $\psi_{E}(x)$ of $H$

$$
\left(-\frac{1}{2 m} \partial_{x}^{2}+V(x)\right) \psi_{E}(x)=E \psi_{E}(x), \quad E \in \mathbb{R}^{+}
$$




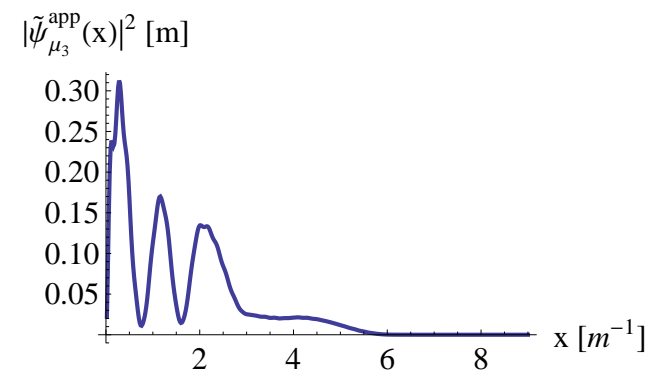

Figure 1: Spatial density of the third resonance $\mu_{3}$.

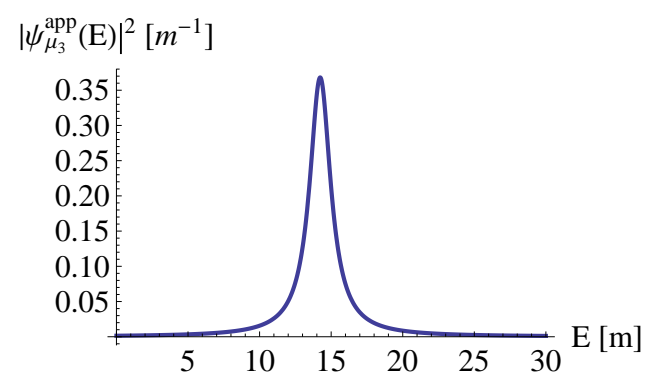

Figure 2: Energy density of the third resonance $\mu_{3}$.

Imposing appropriate boundary conditions we find that

$$
\psi_{E}(x)= \begin{cases}\alpha_{1}(k) \sin k x, & 0<x \leq a, \\ \alpha_{2}(k) e^{i k^{\prime} x}+\beta_{2}(k) e^{-i k^{\prime} x}, & a<x<b, \\ \alpha_{3}(k) e^{i k x}+\beta_{3}(k) e^{-i k x}, & b \leq x,\end{cases}
$$

where $k=\sqrt{2 m E}$ and $k^{\prime}=\sqrt{2 m\left(E-V_{0}\right)}$ for $E \geq V_{0}>0$ or $k^{\prime}=i \sqrt{2 m V_{0}-E}$ for $V_{0}>E \geq 0$. The coefficients in Eq. (29) are given by [16]

$$
\begin{aligned}
\alpha_{2}(k) & =\frac{1}{2} e^{-i k^{\prime} a}\left(\sin k a+\frac{k}{i k^{\prime}} \cos k a\right) \alpha_{1}(k), \\
\beta_{2}(k) & =\frac{1}{2} e^{i k^{\prime} a}\left(\sin k a-\frac{k}{i k^{\prime}} \cos k a\right) \alpha_{1}(k), \\
\alpha_{3}(k) & =\frac{1}{4} e^{-i k b}\left(\left(1+\frac{k^{\prime}}{k}\right) e^{i k^{\prime}(b-a)}\left(\sin k a+\frac{k}{i k^{\prime}} \cos k a\right)\right. \\
& \left.+\left(1-\frac{k^{\prime}}{k}\right) e^{-i k^{\prime}(b-a)}\left(\sin k a-\frac{k}{i k^{\prime}} \cos k a\right)\right) \alpha_{1}(k), \\
\beta_{3}(k) & =\alpha_{3}^{*}(k),
\end{aligned}
$$

where $\alpha_{1}(k)$ is to be determined by normalization conditions.

Given the full set of solutions $\left\{\psi_{E}(x)\right\}_{E \in \mathbb{R}^{+}}$for the continuous energy spectrum one can find the sets $\left\{\psi_{E}^{ \pm}\right\}_{E \in \mathbb{R}^{+}}$of solutions of the Lippmann-Schwinger equation corresponding to incoming and outgoing asymptotic conditions. We have

$$
\psi_{E}^{+}(x):=\left\langle x \mid E^{+}\right\rangle=\frac{i}{2 \alpha_{3}^{*}(k)} \psi_{E}(x), \quad \psi_{E}^{-}(x):=\left\langle x \mid E^{-}\right\rangle=\frac{1}{2 i \alpha_{3}(k)} \psi_{E}(x) .
$$

where $\psi_{E}^{+}(x)$ and $\psi_{E}^{-}(x)$ are, respectively, the incoming and outgoing Lippmann-Schwinger solutions. The normalization conditions for the Lippmann-Schwinger states in Eq. (31) give us $\alpha_{1}(k)=(2 \pi k)^{-1 / 2}$. In the energy representation the $S$-matrix is given by

$$
\tilde{\mathcal{S}}(E)=-\frac{\alpha_{3}(k)}{\alpha_{3}^{*}(k)}
$$

The above expression for the $S$-matrix leads to the calculation of the scattering resonances of the problem. For a resonance point $z=\mu_{j}$ in the lower half-plane below the positive real axis we set $\mu_{j}=E_{\mu_{j}}-i \Gamma_{\mu_{j}} / 2$ with $E_{\mu_{j}}>0$ the resonance energy and $\Gamma_{\mu_{j}}>0$ the resonance width.

For barrier parameters $a=2 m^{-1}, b=3 m^{-1}$, and $V_{0}=5 m$ the three lowest energy resonance poles are given by $\mu_{1} \simeq 0.9106 m-i 0.0012 m, \mu_{2} \simeq 3.5119 m-i 0.0282 m$ and $\mu_{3}=7.1168 m-i 0.4462 m$. We shall focus on the third resonance pole $\mu_{3}$. Utilizing the outgoing Lippmann-Schwinger eigenfunctions $\psi_{E}^{-}(x)$, given by Eqs. (29) 31), the spatial wave function of the approximate resonance state $\psi_{\mu_{3}}^{a p p}(x)$, and its energy density, can be calculated numerically. The probability density $\left|\psi_{\mu_{3}}^{a p p}(x)\right|^{2}$ is shown in Fig. 1 (see [9]), while the energy density $\left|\psi_{\mu_{3}}^{a p p}(E)\right|^{2}$ is shown in Fig. 2. 


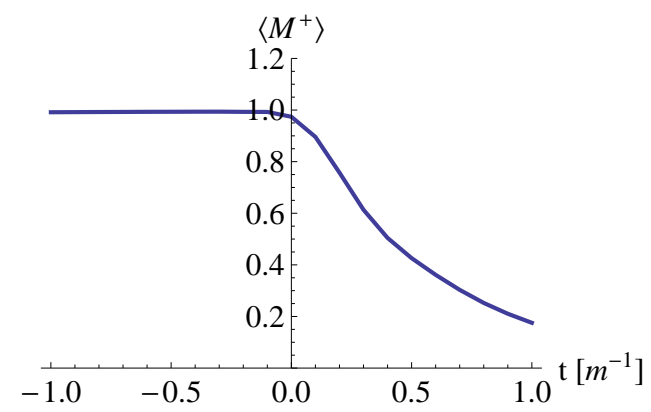

Figure 3: Monotonic decrease of the expectation value of $M^{+}$in the state $\left|\tilde{\psi}_{\mu_{3}}^{a p p}(t)\right\rangle$. Note the transition to exponential decay for $t>0$.

Now apply the outgoing transition decomposition of Eq. (26) to the evolution of the approximate resonance state $\left|\psi_{\mu_{3}}^{a p p}(t)\right\rangle=U(t)\left|\psi_{\mu_{3}}^{a p p}\right\rangle$. According to Eq. (26) the spatial probability distribution of $\left|\psi_{\mu_{3}}^{a p p}(x, t)\right|^{2}$ decomposes into three components

$$
\left|\tilde{\psi}_{\mu_{3}}^{a p p}(x, t)\right|^{2}=\left|\tilde{\psi}_{\mu_{3}, b}^{a p p,+}(x, t)\right|^{2}+2 \operatorname{Re}\left(\left(\tilde{\psi}_{\mu_{3}, b}^{a p p,+}(x, t)\right)^{*} \tilde{\psi}_{\mu_{3}, f}^{a p p,+}(x, t)\right)+\left|\tilde{\psi}_{\mu_{3}, f}^{a p p,+}(x, t)\right|^{2} .
$$

The right-hand side of Eq. [33) is the outgoing transition decomposition of the position probability density of $\psi_{\mu_{3}}^{a p p}(t)$. The first term on the right hand side of Eq. (33) is the backward asymptotic component, the second term is the transient component, and the third term is the forward asymptotic component.

Fig. 4 presents the results of the application of the outgoing transition decomposition to the evolution of the approximate resonance state $\left|\psi_{\mu_{3}}^{a p p}\right\rangle$. Each row represents a 'snapshot' corresponding to a particular time $t$. The left graph in each row shows $\left|\tilde{\psi}_{\mu_{3}}^{a p p}(x, t)\right|^{2}$ at time $t$. The middle graph shows the contribution of its backward asymptotic component $\left|\tilde{\psi}_{\mu_{3}, b}^{a p p,+}(x, t)\right|^{2}$ at time $t$, and the right graph in each row shows the sum of transient and forward asymptotic components $2 \operatorname{Re}\left(\left(\tilde{\psi}_{\mu_{3}, b}^{a p p,+}(x, t)\right)^{*} \tilde{\psi}_{\mu_{3}, f}^{a p p,+}(x, t)\right)+\left|\tilde{\psi}_{\mu_{3}, f}^{a p p}(x, t)\right|^{2}$. It is clearly seen from this sequence of snapshots that the formation phase of the resonance, starting at the negative time asymptote $t \rightarrow-\infty$ and following through the scattering process up to $t=0$, is captured by the backward asymptotic component (the middle column of graphs), while through this whole time interval the contribution of the transient and forward asymptotic components is small. In the decay phase of the resonance, commencing at $t>0$, the middle column of graphs essentially shows the spatial probability density profile of a resonance state, multiplied by an exponentially decaying factor $\exp \left(-\Gamma_{\mu_{3}} t\right)$, which gives the decay of the resonance over time. As the resonance state decays the probability is transferred to the forward asymptotic term, a process captured in the right most column of graphs, and is eventually carried to spatial infinity as the scattering process evolves further towards the forward time asymptote $t \rightarrow \infty$. Observe also that the graphs on the right column in Fig. 4 obtain also negative values. This is due to the contribution of the transient term which is not necessarily positive.

Further understanding of the behavior of the probability density $\left|\tilde{\psi}_{\mu_{3}, b}^{a p p,+}(x, t)\right|^{2}$ may be gained by integrating it over $x$. In this way we get

$$
\begin{aligned}
& \int_{0}^{\infty} d x\left|\tilde{\psi}_{\mu_{3}, b}^{a p p,+}(x, t)\right|^{2}=\int_{0}^{\infty} d x\left\langle\tilde{\psi}_{\mu_{3}, b}^{a p p,+}(t) \mid x\right\rangle\left\langle x \mid \tilde{\psi}_{\mu_{3}, b}^{\text {app },+}(t)\right\rangle \\
= & \int_{0}^{\infty} d x\left\langle\Lambda^{+} \tilde{\psi}_{\mu_{3}}^{a p p}(t) \mid x\right\rangle\left\langle x \mid \Lambda^{+} \tilde{\psi}_{\mu_{3}}^{a p p}(t)\right\rangle=\left\langle\tilde{\psi}_{\mu_{3}}^{a p p}(t)\left|M^{+}\right| \tilde{\psi}_{\mu_{3}}^{a p p}(t)\right\rangle,
\end{aligned}
$$

which is just the expectation value of the Lyapunov operator $M^{+}$in the state $\left|\psi_{\mu_{3}}^{a p p}(t)\right\rangle$. A plot of this behavior is presented in Fig. 3. The decay of the expectation value of $M^{+}$matches the resonance $\operatorname{decay}$ factor $\exp \left(-\Gamma_{\mu_{3}} t\right)$ mentioned above. 

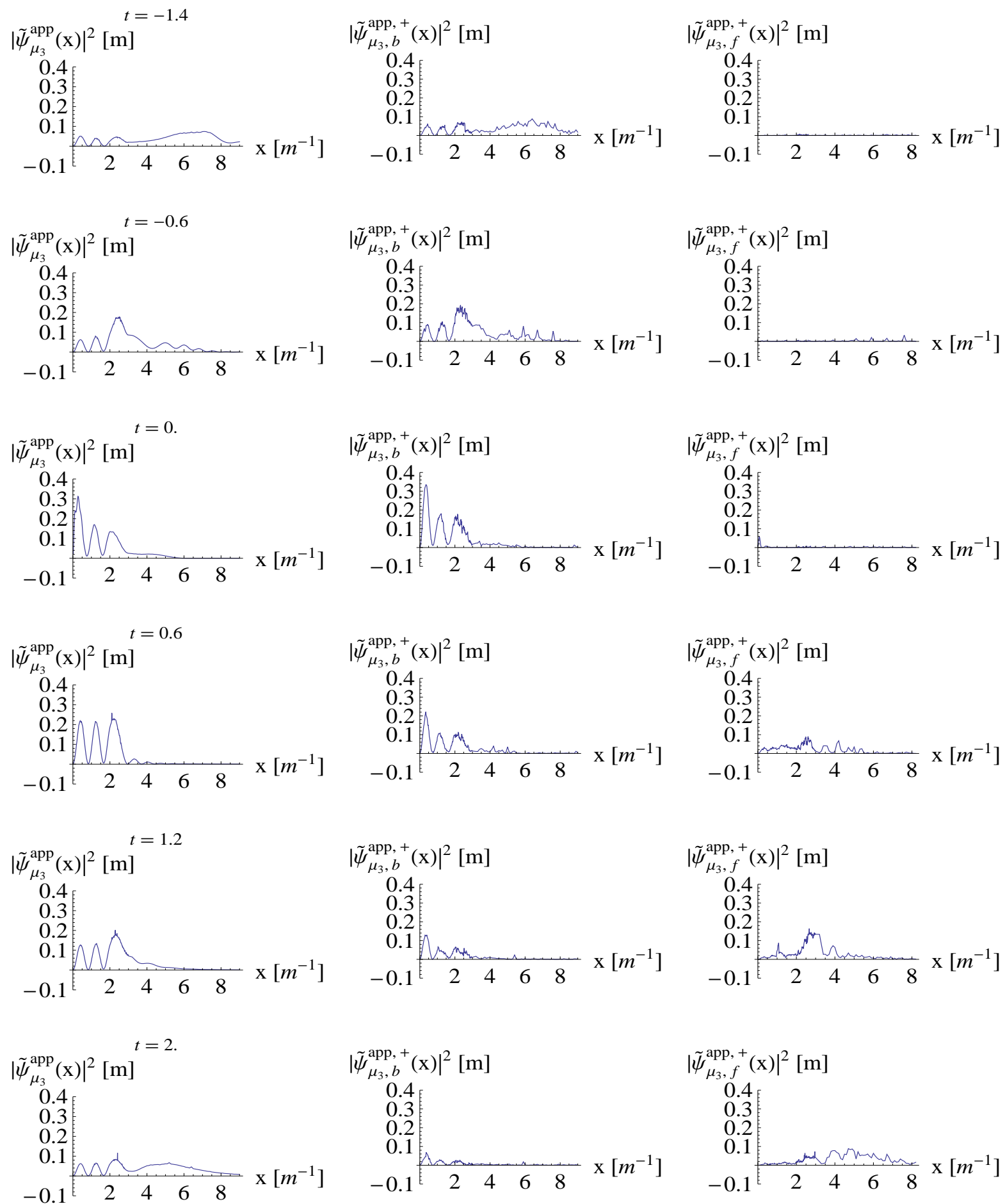

Figure 4: Transition decomposition of $\psi_{\mu_{3}}^{a p p}(t)$. Each row of graphs represents a time frame at time $t$ indicated on the left row. The left row depicts $\left|\psi_{\mu_{3}}^{a p p}(x, t)\right|^{2}$, the middle row depicts $\left|\psi_{\mu_{3}, b}^{a p p,}{ }^{+}(x, t)\right|^{2}$, while the right row depicts $\left|\psi_{\mu_{3}, f}^{a p p,+}(x, t)\right|^{2}$. 


\section{Acknowledgments}

Y. Strauss, and J. Silman and S. Machnes, acknowledge support from the ISF (Grants. 1169/06 and 784/06, respectively). J. Silman also acknowledges the support of the Inter-University Attraction Poles Programme (Belgian Science Policy) under Project IAP-P6/10 (Photonics@be) and of the FNRS.

[1] A.S. Holevo, Probabilistic and Statistical Aspects of Quantum Theory (North-Holland, 1982).

[2] P. Busch, M. Grabowski, and P.J. Lahti, Operational Quantum Physics (Springer 1995).

[3] W.E. Pauli, in Handbuch der Physik, (Springer-Verlag, 1926).

[4] See, for example, G.W. Mackey, The Theory of Unitary Group Representations (University of Chicago Press, 1976).

[5] Y. Strauss, J. Math. Phys. 51, 022104 (2010).

[6] Y. Strauss, J. Silman, S. Machnes, and L.P. Horwitz, arXiv:1101.3969 [quant-ph]. See also Y. Strauss, J. Silman, S. Machnes, and L.P. Horwitz, arXiv:0802.2448 [quant-ph].

[7] G.C. Hegerfeldt and J.G. Muga, arXiv:1008.4731 [quant-ph].

[8] Y. Strauss, J. Math. Phys. 46, 102109 (2005).

[9] Y. Strauss, L.P. Horwitz, and A.J. Volovick, Math. Phys. 47. 123505 (2006).

[10] P.D. Lax and R.S. Phillips, Scattering Theory (Academic Press, 1967).

[11] Y. Strauss, L.P. Horwitz, and E. Eisenberg, J. Math. Phys. 41, 8050 (2000).

[12] Y. Strauss, Int. J. Theor. Phys. 42, 2285 (2003).

[13] L.P. Horwitz and J.-P. Marchand, Rocky Mt. J. Math. 1, 225 (1971)

[14] B. Misra, E.C.G. Sudarshan, J. Math. Phys. 18, 756 (1977).

[15] Y. Strauss, J. Math. Phys. 46, 032104 (2005).

[16] R. de la Madrid and M. Gadella, Amer. J. Phys 70, 626 (2002). 